\title{
A Modified 3-Point Adams Block Method of the Variable Step Size Strategy for Solving Neutral Delay Differential Equations
}

\author{
Ubaidullah Yashkun ${ }^{1,2}$, Nurul Huda Abdul Aziz ${ }^{1}$
}

\begin{abstract}
:
A modified 3-point Adams block method of order six (3ABM6) to solve neutral delay differential equations (NDDEs) using variable step size strategy is developed. The approximate solution of the retarded $y(x-\tau)$ and the neutral terms $y^{\prime}(x-\tau)$ of the neutral delay differential equations at the grid points is obtained using the Newton divided difference interpolation technique. The proposed method will approximate the solution in each step using the three-points concurrently. To determine the performance of the proposed method, the maximum errors (MAXERR) and total number of function calls (FNC) will be compared with the method of 2-point order six predictor-corrector. The numerical results show that the $3 \mathrm{ABM} 6$ reduces the number of function calls and better accuracy in term of MAXERR.
\end{abstract}

Keywords: Neutral Delay Differential Equations, Adams Block Method, Newton Interpolation Technique, Variable Step Size Technique.

\section{Introduction}

In the fields of Science and Technology, the ordinary differential equations (ODEs) can be used to formulate the real-life problems by using initial value problem (IVP).

$$
\frac{d y}{d x}=f(x, y) \quad a \leq x \leq b
$$

Where, the function $y$ usually represents physical quantities that evolve over time. Moreover, the delay differential equations (DDEs) are applied extensively in engineering, particularly the delay differential equations of the neutral type that usually arise in many scientific areas due to their ability to model many real-life phenomena. Neutral Delay Differential Equations do not depend only on the history of the function $y(x-\tau)$ formulated but also on the history of the function derivative $y^{\prime}(x-\tau)$.

$$
\begin{array}{lr}
y^{\prime}(x)=f\left(x, y(x), y(x-\tau(x)), y^{\prime}(x), y^{\prime}\left(x-\tau^{*}(x)\right)\right) & x \geq x_{0}, \\
y(x)=\phi(x) & \tau \leq x \leq x_{0},
\end{array}
$$

Where, $\tau(x)>0$ and $\tau^{*}(x)>0$ are the delay arguments, and $\phi(x)$ is the initial value function.

Several algorithms are envisaged in the literature to approximate the results of Neutral Delay Differential Equation (NDDEs) of type (2) which is $y^{\prime}(x)$ having $f$ as an independent function. Neves [1], Bellen and Zennaro[2] and AlMutib[3] discussed algorithms based on one-step methods, while Jackiewicz and Lo[4] and Tavernini[5] discussed algorithms based on liner multistep methods. Fabiano and Payne[6] extended the spline approximation technique for Neutral Delay Differential Equations. The continuous Galerkin finite element method

\footnotetext{
${ }^{I}$ Institute of Engineering Mathematics, Universiti Malaysia Perlis, Kampus Pauh Putra, 02600, Arau, Perlis,Malaysia

${ }^{2}$ Sukkur IBA University, Airport Road, Sukkur, 65200, Sindh, Pakistan.

Corresponding Author: ubaidullah@iba-suk.edu.pk SJCMS | P-ISSN: 2520-0755| E-ISSN: 2522-3003 @ 2019 Sukkur IBA University - All Rights Reserved
} 
is used by Qin et al. [7] to solve the Linear Delay Differential Equations.

Wen et al. [8] discussed the numerical stability of NDDEs and Baker et al. [9] discussed the role of NDDEs in cell growth phenomena. The Neutral Functional Differential Equations (NFDEs) numerical solution is studied by $\mathrm{Hu}$ et al. [10] using linear multistep methods (LMM). The analytical and numerical stability of the nonlinear neutral delay-integral differential equations was studied by $\mathrm{P}$. $\mathrm{Hu}$ and $\mathrm{C}$. Huang [11]. An approximate solution for NFNDEs was also obtained by Wang and Li [12] using numerical methods, namely the one-leg $\theta$ methods.

The Adams Block Methods (ABM) are efficient and computational cost-effective methods compared to the other numerical methods. Seong and Majid[13] studied direct two point order four and five multistep block method to solve the second order delay differential equations (DDEs). In their study the formulation and stability of the block method is discussed. Aziz et al. [14] presented the delay differential equations (DDEs) numerical solution using a predictor-corrector scheme in modified block method. ABM will compute the $y$ approximate values in the block corresponding to the independent values of $x$. The one-block r-point technique for the 2 nd order initial value problem is proposed by Fatunla [15]. A block method will compute simultaneously the solution values at numerous different points on the $x$-axis of the blocks by Ishak et al. [16] and Alkasassbeh and Omar [17].

In this paper, we introduce a new modified 3-point Adams block method of order six for first-order NDDEs. Some numerical results are shown in the tables for the proposed method. Here is the order of this paper. Section 2 formulation of the method is presented. In section 3, order, consistency and zero stability are discussed. In section 4 implementation of retarded and neutral terms are discussed. In section 5, the strategy of variable step size is discussed. Section 6, presents the algorithm of the method. Section 7, presents the numerical results, while discussions and conclusion are given in section 8 .

\section{The formulation of the 3-point Adams block method}

Consider the first-order NDDEs (2) and discretize the interval $[a, b]$ to subintervals

$x_{n-4}, x_{n-3}, \ldots, x_{n-4+k}, k=0,1,2, \ldots$

such that $a=x_{n-4}$ and $b=x_{n-4+k}$. From Fig. 1, the proposed method is used to evaluate $\quad y_{n+1}, y_{n+2}$ and $y_{n+3}$ corresponding to the grid values $x_{n+1}, x_{n+2}$ and $x_{n+3}$ having the variable step size $\boldsymbol{h}$ respectively. The four initial values $y_{n-3}, y_{n-2}, y_{n-1}$ and $y_{n}$ are obtained using the Euler method with the initial conditions corresponding to the values $x_{n-3}, x_{n-2}, x_{n-1}$ and $x_{n}$ having step ratios $r h$ and $q h$.

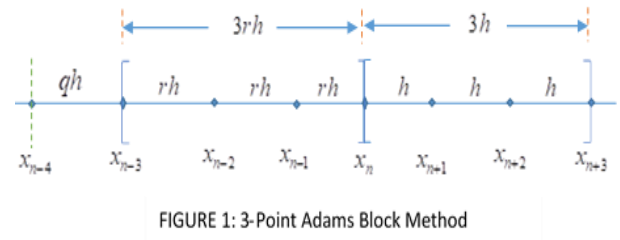

Source: Author's own created

$y_{n+1}$ can be determine by integrating (1) as;

$$
y\left(x_{n+1}\right)=y\left(x_{n}\right)+\int_{x_{n}}^{x_{n+1}} f(x, y) d x
$$

In (3), to integrate the function $f(x, y)$, a Lagrange interpolation is substituted with the function. The approximation of $y_{n+1}$ by using Maple with the ratio $r$ is as follows:

$$
y\left(x_{n+1}\right)=y\left(x_{n}\right)+\int_{x_{n}}^{x_{n+1}} P_{4}(x) d x
$$

By substitution

$$
s=\frac{x-x_{n}}{h} \text { and } s=\frac{x-x_{n+3}}{h}
$$


$\mathrm{ABM}$ of order six for the variable step size For the Corrector when $r=1$ at the limit ratios is as follows:

points -3 to $-2,-2$ to -1 and -1 to O

$$
\begin{aligned}
y_{n+1}=y_{n} & +h \int_{-3}^{-2} \frac{1}{120}(s+5)(s+4)(s+3)(s+2)(s+1) d s f_{n+3} \\
& -h \int_{-3}^{-2} \frac{1}{24}(s+5)(s+4)(s+3)(s+2)(s) d s f_{n+2} \\
& +h \int_{-3}^{-2} \frac{1}{12}(s+5)(s+4)(s+3)(s+1)(s) d s f_{n+1} \\
& -h \int_{-3}^{-2} \frac{1}{12}(s+5)(s+4)(s+2)(s+1)(s) d s f_{n} \\
& +h \int_{-3}^{-2} \frac{1}{24}(s+5)(s+3)(s+2)(s+1)(s) d s f_{n-1} \\
& -h \int_{-3}^{-2} \frac{1}{120}(s+4)(s+3)(s+2)(s+1)(s) d s * f_{n-2} \\
y_{n+1}=y_{n}+ & \frac{h}{1440}\left(11 f_{n+3}-93 f_{n+2}+802 f_{n+1}+802 f_{n}-93 f_{n-1}+11 f_{n-2}\right) \\
y_{n+2}=y_{n+1}+ & \frac{h}{1440}\left(-27 f_{n+3}+637 f_{n+2}+1022 f_{n+1}-258 f_{n}+77 f_{n-1}-11 f_{n-2}\right) \\
y_{n+3}=y_{n+2}+ & \frac{h}{1440}\left(475 f_{n+3}+1427 f_{n+2}-798 f_{n+1}+482 f_{n}-173 f_{n-1}+27 f_{n-2}\right)
\end{aligned}
$$

For the corrector when $r=2$ at the limit points -3 to $-2,-2$ to $-\mathbf{1}$ and $-\mathbf{1}$ to $\mathrm{O}$

$$
\begin{aligned}
& y_{n+1}=y_{n}+\frac{h}{100800}\left(1328 f_{n+3}-9765 f_{n+2}+63952 f_{n+1}+46375 f_{n}-1183 f_{n-1}+93 f_{n-2}\right) \\
& y_{n+2}=y_{n+1}+\frac{h}{100800}\left(-2352 f_{n+3}+47285 f_{n+2}+65072 f_{n+1}-9975 f_{n}+847 f_{n-1}-77 f_{n-2}\right) \\
& y_{n+3}=y_{n+2}+\frac{h}{100800}\left(34288 f_{n+3}+93835 f_{n+2}-41328 f_{n+1}+15575 f_{n}-1743 f_{n-1}+173 f_{n-2}\right)
\end{aligned}
$$

For the corrector when $r=\frac{1}{2}$ at the limit points -3 to $-2,-2$ to -1 and -1 to $\mathrm{O}$

$$
\begin{aligned}
& y_{n+1}=y_{n}+\frac{h}{2520}\left(11 f_{n+3}-105 f_{n+2}+1211 f_{n+1}+1981 f_{n}-704 f_{n-1}+126 f_{n-2}\right) \\
& y_{n+2}=y_{n+1}+\frac{h}{2520}\left(-39 f_{n+3}+1057 f_{n+2}+1981 f_{n+1}-1029 f_{n}+704 f_{n-1}-154 f_{n-2}\right) \\
& y_{n+3}=y_{n+2}+\frac{h}{2520}\left(8011 f_{n+3}+2639 f_{n+2}-1869 f_{n+1}+2261 f_{n}-1728 f_{n-1}+406 f_{n-2}\right)
\end{aligned}
$$

The similar procedure can be used to get the one less order of predictor formulas. 
3. Order Consistency and Zero Stability

In this study, the order of corrector is one order higher than the predictor. J. D. Lambert [18] gives the following definitions of order, consistency and zero-stability for the linear multistep methods. The order of this new developed 3ABM6 method is determined based on [18] and [13].

Theorem 1 (Lambert [18]). The general multistep method;

$\sum_{m=0}^{s} \alpha_{m} y_{n+m}=h \sum_{m=0}^{s} \beta_{m} f\left(x_{n+m}, y_{n+m}\right)$
The order $p \geq 1$ if and only if

$C_{0}=\sum_{m=0}^{s} \alpha_{m}=0$,

$C_{P}=\sum_{m=0}^{s} \frac{m^{k}}{k !} \alpha_{m}-\sum_{m=0}^{s} \frac{m^{k-1}}{(k-1) !} \beta_{m}=0, k=1,2, \ldots, p$,

$C_{p+1}=\sum_{m=0}^{s} \frac{m^{p+1}}{(p+1) !} \alpha_{m}-\sum_{m=0}^{s} \frac{m^{p}}{p !} \beta_{m} \neq 0$.

The three-point block method can be written as matrix difference equation.

$$
\alpha_{m} Y_{n+m}=h \beta_{m} f_{n+m}
$$

Where,

$$
\begin{gathered}
\alpha_{m}=\left[\begin{array}{rrrrrr}
0 & 0 & -1 & 1 & 0 & 0 \\
0 & 0 & 0 & -1 & 1 & 0 \\
0 & 0 & 0 & 0 & -1 & 1
\end{array}\right], \quad \beta_{m}=\frac{1}{1440}\left[\begin{array}{rrrrrr}
11 & -93 & 802 & 802 & -93 & 11 \\
-11 & 77 & -258 & 1022 & 637 & -27 \\
27 & -173 & 482 & -798 & 1427 & 475
\end{array}\right], \\
Y_{n+m}=\left[\begin{array}{llllll}
y_{n-2} & y_{n-1} & y_{n} & y_{n+1} & y_{n+2} & y_{n+3}
\end{array}\right]^{T} \\
\text { and } \\
f_{n+m}=\left[\begin{array}{llllll}
f_{n-2} & f_{n-1} & f_{n} & f_{n+1} & f_{n+2} & f_{n+3}
\end{array}\right]^{T}
\end{gathered}
$$

From Theorem 1, the order of the corrector formulae of Equation (6) is

$$
\begin{aligned}
& C_{\mathrm{o}}=\left[\begin{array}{l}
\mathrm{O} \\
\mathrm{O} \\
\mathrm{O}
\end{array}\right]+\left[\begin{array}{l}
\mathrm{O} \\
\mathrm{O} \\
\mathrm{O}
\end{array}\right]+\left[\begin{array}{r}
-1 \\
\mathrm{O} \\
\mathrm{O}
\end{array}\right]+\left[\begin{array}{r}
1 \\
-1 \\
\mathrm{O}
\end{array}\right]+\left[\begin{array}{r}
0 \\
1 \\
-1
\end{array}\right]+\left[\begin{array}{l}
\mathrm{O} \\
\mathrm{O} \\
1
\end{array}\right]=\left[\begin{array}{l}
\mathrm{O} \\
\mathrm{O} \\
\mathrm{O}
\end{array}\right] \\
& C_{1}=\alpha_{1}+2 \alpha_{2}+3 \alpha_{3}+4 \alpha_{4}+5 \alpha_{5}+6 \alpha_{6}-\left(\beta_{0}+\beta_{1}+\beta_{2}+\beta_{3}+\beta_{4}+\beta_{5}+\beta_{6}\right) \\
& C_{1}=\left[\begin{array}{l}
0 \\
0 \\
0
\end{array}\right]+2\left[\begin{array}{r}
-1 \\
0 \\
0
\end{array}\right]+3\left[\begin{array}{r}
1 \\
-1 \\
0
\end{array}\right]+4\left[\begin{array}{r}
0 \\
1 \\
-1
\end{array}\right]+5\left[\begin{array}{l}
0 \\
0 \\
1
\end{array}\right]+6\left[\begin{array}{l}
0 \\
0 \\
0
\end{array}\right] \\
& -\frac{1}{1440}\left(\left[\begin{array}{r}
11 \\
-11 \\
27
\end{array}\right]+\left[\begin{array}{r}
-93 \\
77 \\
-173
\end{array}\right]+\left[\begin{array}{r}
802 \\
-258 \\
482
\end{array}\right]+\left[\begin{array}{r}
802 \\
1022 \\
-798
\end{array}\right]+\left[\begin{array}{r}
-93 \\
637 \\
1427
\end{array}\right]+\left[\begin{array}{r}
11 \\
-27 \\
475
\end{array}\right]+\left[\begin{array}{l}
0 \\
0 \\
0
\end{array}\right]\right) \\
& C_{1}=\left[\begin{array}{l}
0 \\
0 \\
0
\end{array}\right] \\
& C_{2}=\frac{1}{2 !} \alpha_{1}+\frac{2^{2}}{2 !} \alpha_{2}+\frac{3^{2}}{2 !} \alpha_{3}+\frac{4^{2}}{2 !} \alpha_{4}+\frac{5^{2}}{2 !} \alpha_{5}+\frac{6^{2}}{2 !} \alpha_{6}-\left(\beta_{1}+2 \beta_{2}+3 \beta_{3}+4 \beta_{4}+5 \beta_{5}+6 \beta_{6}\right) \\
& C_{2}=\frac{1}{2 !}\left[\begin{array}{l}
0 \\
0 \\
0
\end{array}\right]+\frac{4}{2 !}\left[\begin{array}{r}
-1 \\
0 \\
0
\end{array}\right]+\frac{9}{2 !}\left[\begin{array}{r}
1 \\
-1 \\
0
\end{array}\right]+\frac{16}{2 !}\left[\begin{array}{r}
0 \\
1 \\
-1
\end{array}\right]+\frac{25}{2 !}\left[\begin{array}{l}
0 \\
0 \\
1
\end{array}\right]+\frac{36}{2 !}\left[\begin{array}{l}
0 \\
0 \\
0
\end{array}\right] \\
& -\frac{1}{1440}\left(\left[\begin{array}{c}
-93 \\
77 \\
-173
\end{array}\right]+2\left[\begin{array}{c}
802 \\
-258 \\
482
\end{array}\right]+3\left[\begin{array}{c}
802 \\
1022 \\
-798
\end{array}\right]+4\left[\begin{array}{c}
-93 \\
637 \\
1427
\end{array}\right]+5\left[\begin{array}{c}
11 \\
-27 \\
475
\end{array}\right]+6\left[\begin{array}{l}
0 \\
0 \\
0
\end{array}\right]\right) \\
& C_{2}=\left[\begin{array}{l}
\mathrm{O} \\
\mathrm{O} \\
\mathrm{O}
\end{array}\right] \\
& C_{3}=\left[\begin{array}{lll}
\mathrm{O} & \mathrm{O} & \mathrm{o}
\end{array}\right]^{T}, C_{4}=\left[\begin{array}{lll}
\mathrm{O} & \mathrm{O} & \mathrm{o}
\end{array}\right]^{T}, C_{5}=\left[\begin{array}{lll}
\mathrm{O} & \mathrm{O} & \mathrm{o}
\end{array}\right]^{T}, C_{6}=\left[\begin{array}{lll}
\mathrm{O} & \mathrm{O} & \mathrm{o}
\end{array}\right]^{T}
\end{aligned}
$$

Sukkur IBA Journal of Computing and Mathematical Science - SJCMS | Vol. 3 No. 2 July - December 2019 ๑ Sukkur IBA University 


$$
\begin{aligned}
& C_{7}=\frac{1}{7 !} \alpha_{1}+\frac{2^{7}}{7 !} \alpha_{2}+\frac{3^{7}}{7 !} \alpha_{3}+\frac{4^{7}}{7 !} \alpha_{4}+\frac{5^{7}}{7 !} \alpha_{5}+\frac{6^{7}}{7 !} \alpha_{6} \\
& -\left(\frac{1}{6 !} \beta_{1}+\frac{2^{6}}{6 !} \beta_{2}+\frac{3^{6}}{6 !} \beta_{3}+\frac{4^{6}}{6 !} \beta_{4}+\frac{5^{6}}{6 !} \beta_{5}+\frac{6^{6}}{6 !} \beta_{6}\right) \\
& C_{7}=\frac{1}{7 !}\left[\begin{array}{l}
0 \\
0 \\
0
\end{array}\right]+\frac{2^{7}}{7 !}\left[\begin{array}{c}
-1 \\
0 \\
0
\end{array}\right]+\frac{3^{7}}{7 !}\left[\begin{array}{r}
1 \\
-1 \\
0
\end{array}\right]+\frac{4^{7}}{7 !}\left[\begin{array}{r}
0 \\
1 \\
-1
\end{array}\right]+\frac{5^{7}}{7 !}\left[\begin{array}{l}
0 \\
0 \\
1
\end{array}\right]+\frac{6^{7}}{7 !}\left[\begin{array}{l}
0 \\
0 \\
0
\end{array}\right] \\
& -\frac{1}{1440}\left(\frac{1}{6 !}\left[\begin{array}{c}
-93 \\
77 \\
-173
\end{array}\right]+\frac{2^{6}}{6 !}\left[\begin{array}{r}
802 \\
-258 \\
482
\end{array}\right]+\frac{3^{6}}{6 !}\left[\begin{array}{c}
802 \\
1022 \\
-798
\end{array}\right]+\frac{4^{6}}{6 !}\left[\begin{array}{r}
-93 \\
637 \\
1427
\end{array}\right]+\frac{5^{6}}{6 !}\left[\begin{array}{c}
11 \\
-27 \\
475
\end{array}\right]+\frac{6^{6}}{6 !}\left[\begin{array}{l}
0 \\
0 \\
0
\end{array}\right]\right) \\
& C_{7}=\left[\begin{array}{c}
\frac{-191}{60480} \\
\frac{271}{60480} \\
\frac{-863}{60480}
\end{array}\right] \neq 0 .
\end{aligned}
$$

Equation (12) implies that $C_{7} \neq 0$ therefore, the order of the proposed method is order six.

Definition 2. The LMM in (9) is referred to as consistent such that order $p \geq 1$. The characteristics polynomials of LMM (9) are

$$
\begin{aligned}
& \rho(\xi)=\sum_{i=0}^{k} A_{i}^{*} \xi^{i}, \\
& \sigma(\xi)=\sum_{i=0}^{k} B_{i}^{*} \xi^{i} .
\end{aligned}
$$

The LMM is consistent iff $\rho(1)=0$ and $\rho^{\prime}(1)=\sigma(1)$

Definition 3. Let the $\operatorname{root}|\xi|=1$ for the polynomial $\rho(\xi)$, then the LMM (13) is referred to as zero-stable.

\section{Implementation of Retarded and Neutral Term}

The first order NDDEs approximate solution depends on the retarded $y(t-\tau)$ and neutral $y^{\prime}(t-\tau)$ terms. The delay of these terms may be constant $(t-\tau)$, time dependent $(\tau(t))$ or state dependent $(\tau(t, X(t)))$. In this paper, the Newton divided difference technique is used to approximate these terms. For implementation, the required points are:

$$
\begin{aligned}
& \left(x_{n-4}, y_{n-4}\right),\left(x_{n-3}, y_{n-3}\right),\left(x_{n-2}, y_{n-2}\right),\left(x_{n-1}, y_{n-1}\right), \\
& \left(x_{n}, y_{n}\right),\left(x_{n+1}, y_{n+1}\right),\left(x_{n+2}, y_{n+2}\right) \text { and }\left(x_{n+3}, y_{n+3}\right)
\end{aligned}
$$

The following recursion relation will be used for Newton divided differences.

$$
f\left[x_{n-4}, x_{n-3}, \ldots, x_{n-4+k}\right]=\frac{f\left[x_{n-3}, x_{n-2}, \ldots, x_{n-4+k}\right]-f\left[x_{n-4}, x_{n-3}, \ldots, x_{n-5+k}\right]}{x_{n-4+k}-x_{n-4}}
$$

$$
k=0,1,2 \ldots
$$

\section{The Strategy of Variable Step- Size}

control strategy will be like, the next step size is limited to double, as the previous step size when the computation proceeds as studied by [19]. The variable step size The variable step size strategy is recommended for this study. Step-size $\boldsymbol{h}$ strategy may reduce the formulae that need 
to be stored in the code, such that the storage capacity will increase. The evaluation technique

is $P(E C)^{s}$ or $P(E C)^{s} E$, such that $P$ represents the predictor, $E$ represents evaluation, $C$ represents the corrector and $S$ is the number of iterations. The $P(E C)^{s} E$ mode is used if we need to calculate the function value of $f_{n+i}^{(s)}=f\left(x_{n+i}, y_{n+i}^{(s)}\right)$ otherwise we use $P(E C)^{s}$ mode. Modified 3-point Adams block method of order six varies the step size $h$, to obtain three values during an integration step. Therefore, the efficiency of the method is acquired by taking the most optimal step size while reaching the desired accuracy.

\section{Algorithm of the 3-point Adams Block Method of Order Six to Solve NDDEs}

Step 1: Set TOL, initial condition $x_{0}, y_{0}$ and step size $\boldsymbol{h}$

Step 2:

$y^{\prime}(x)=f\left(x, y(x), y(x-\tau(x)), y^{\prime}(x), y^{\prime}\left(x-\tau^{*}\left(x_{)}^{x}\right)\right)\right.$

Step 3:

$y(x)=\phi(x), y(0)=1$.

Step 4:

For $i=1,2,3$ and 4 calculate $x-\tau$ for

$y$ and its derivative. If $x-\tau \leq \mathrm{x}_{0}$, go to

3 else calculate $f(x, y)$ from 2 , go to 5

Step 5:

$y_{i}=y_{i-1}+h * f_{i-1}$

Step 6:

For $i=5,6$ and 7 calculate $x-\tau$ for $y$ and its derivative. If $x-\tau \leq \mathrm{x}_{0}$, go to 3 else go to 7

Step 7:

$y\left(x_{n+1}\right)=y\left(x_{n}\right)+\frac{h}{1440} \sum_{i=1}^{6} \mathrm{~A}_{i} f_{n-i+4}$
Step 8: If $\left|y_{n}^{(k+1)}-y_{n}^{k}\right| \leq 1.0 * T O L$ go to

9 else go to 4

Step 9: Stop

\section{Problems and Numerical Results}

In this section, three problems are present which demonstrates the implementation of the modified 3-point Adams block method of order six for first-order neutral delay differential equations (2). The computational results of the problems present confirm the efficiency and accuracy of the method. The analysis of the accuracy and efficiency of the method are based on maximum errors and the number of total steps taken. The approximate results are compared with the results of the 2-point order six predictor-corrector method. The maximum errors and number of total steps taken are plotted in Figs. 2-4.

Problem 1. (Jackiewicz [20])

$y^{\prime}(x)=0.75 x^{2} y(x) /\left(1+x^{2}\right)+y(x / 2)-\left(y^{\prime}((x-1) / 2)\right)^{2}$, $x \in[0,1], y(x)=1+x^{2}$,

$x \in[-0.5,0]$

exact solution

$y(x)=1+x^{2}, x \in[-0.5,1]$

Problem 2. (Jackiewicz [20])

$y^{\prime}(x)=\exp \left(1-2 x^{2}\right) y\left(x^{2}\right)\left(y^{\prime}(x-1 /(1+x))\right)^{1+x}, x \in[0,1]$, $y(x)=\exp (x), x \in[-1,0]$.

The exact solution is

$$
y(x)=\exp (x), x \in[-1,1] \text {. }
$$

Problem 3. (Jackiewicz [20])

$y^{\prime}(x)=1+y(x)-2 y^{2}(x / 2)-y^{\prime}(x-\pi), x \in[0, \pi]$,

$y(x)=\cos (x), x \in[-\pi, 0]$.

The exact solution

$y(x)=\cos (x), x \in[-\pi, \pi]$.

Following abbreviations are used in tables 1-3

TOL: Tolerance Defined

MTD: Employed Method

TS: Total Number of steps

FS: Number of failing steps 
MAXERR: Maximum Error

FCN: Number of function calls
3ABM6: Modified 3-Point Adams block method

N2PC6: Neutral 2-point 6-order

Table I: Approximate results for Problem 1

\begin{tabular}{|l|l|l|l|l|l|l|}
\hline TOL & MTD & TS & FS & FNC & MAXERR & AVERR \\
\hline \multirow{2}{*}{$2^{-2}$} & 3ABM6 & 10 & 0 & 32 & $4.48 \mathrm{E}(-07)$ & $1.86 \mathrm{E}(-07)$ \\
& N2PC6 & 11 & 0 & 39 & $1.42 \mathrm{E}(-05)$ & $2.49 \mathrm{E}(-06)$ \\
\hline \multirow{2}{*}{$2^{-4}$} & 3ABM6 & 14 & 0 & 40 & $4.34 \mathrm{E}(-09)$ & $2.84 \mathrm{E}(-09)$ \\
& N2PC6 & 14 & & 51 & $1.24 \mathrm{E}(-08)$ & $3.17 \mathrm{E}(-09)$ \\
\hline \multirow{2}{*}{$2^{-6}$} & 3ABM6 & 17 & 0 & 46 & $4.34 \mathrm{E}(-11)$ & $2.75 \mathrm{E}(-11)$ \\
& N2PC6 & 18 & 0 & 67 & $1.71 \mathrm{E}(-09)$ & $2.06 \mathrm{E}(-10)$ \\
\hline \multirow{2}{*}{$2^{-8}$} & 3ABM6 & 20 & 0 & 52 & $4.04 \mathrm{E}(-13)$ & $2.68 \mathrm{E}(-13)$ \\
& N2PC6 & 21 & 0 & 80 & $3.23 \mathrm{E}(-13)$ & $2.39 \mathrm{E}(-13)$ \\
\hline \multirow{2}{*}{$2^{-10}$} & 3ABM6 & 24 & 0 & 60 & $2.81 \mathrm{E}(-15)$ & $2.44 \mathrm{E}(-15)$ \\
& N2PC6 & 24 & 0 & 92 & $1.90 \mathrm{E}(-11)$ & $7.37 \mathrm{E}(-13)$ \\
\hline
\end{tabular}

Table II: Approximate results for Problem 2

\begin{tabular}{|c|c|c|c|c|c|c|}
\hline TOL & MTD & TS & FS & FNC & MAXERR & AVERR \\
\hline $2^{-2}$ & 3ABM6 & 10 & 0 & 32 & $3.71 \mathrm{E}(-07)$ & $1.50 \mathrm{E}(-07)$ \\
& N2PC6 & 11 & 0 & 43 & $3.94 \mathrm{E}(-07)$ & $1.41 \mathrm{E}(-07)$ \\
\hline \multirow{2}{*}{$2^{-4}$} & 3ABM6 & 15 & 0 & 42 & $5.54 \mathrm{E}(-07)$ & $7.03 \mathrm{E}(-08)$ \\
& N2PC6 & 14 & 0 & 53 & $2.14 \mathrm{E}(-09)$ & $1.04 \mathrm{E}(-09)$ \\
\hline \multirow{2}{*}{$2^{-6}$} & 3ABM6 & 19 & 0 & 53 & $1.88 \mathrm{E}(-07)$ & $2.34 \mathrm{E}(-08)$ \\
& N2PC6 & 19 & 0 & 68 & $3.27 \mathrm{E}(-09)$ & $3.60 \mathrm{E}(-10)$ \\
\hline \multirow{2}{*}{$2^{-8}$} & 3ABM6 & 30 & 0 & 72 & $3.45 \mathrm{E}(-10)$ & $7.45 \mathrm{E}(-10)$ \\
& N2PC6 & 27 & 0 & 92 & $1.91 \mathrm{E}(-11)$ & $3.33 \mathrm{E}(-12)$ \\
\hline \multirow{2}{*}{$2^{-10}$} & 3ABM6 & 53 & 0 & 118 & $5.60 \mathrm{E}(-11)$ & $1.25 \mathrm{E}(-11)$ \\
& N2PC6 & 33 & 0 & 110 & $6.11 \mathrm{E}(-12)$ & $1.10 \mathrm{E}(-12)$ \\
\hline
\end{tabular}

Table III: Approximate results for Problem 3

\begin{tabular}{|c|c|c|c|c|c|c|}
\hline TOL & MTD & TS & FS & FNC & MAXERR & AVERR \\
\hline \multirow{2}{*}{$2^{-2}$} & 3ABM6 & 14 & 0 & 40 & $3.24 \mathrm{E}(-04)$ & $2.40 \mathrm{E}(-05)$ \\
& N2PC6 & 13 & 0 & 50 & $7.03 \mathrm{E}(-04)$ & $6.41 \mathrm{E}(-05)$ \\
\hline \multirow{2}{*}{$2^{-4}$} & 3ABM6 & 22 & 0 & 57 & $2.92 \mathrm{E}(-05)$ & $1.76 \mathrm{E}(-06)$ \\
& N2PC6 & 17 & & 72 & $2.04 \mathrm{E}(-05)$ & $1.38 \mathrm{E}(-06)$ \\
\hline \multirow{2}{*}{$2^{-6}$} & 3ABM6 & 32 & 0 & 85 & $9.27 \mathrm{E}(-07)$ & $1.54 \mathrm{E}(-07)$ \\
& N2PC6 & 25 & 0 & 106 & $4.73 \mathrm{E}(-07)$ & $4.14 \mathrm{E}(-08)$ \\
\hline \multirow{2}{*}{$2^{-8}$} & 3ABM6 & 50 & 0 & 122 & $2.19 \mathrm{E}(-07)$ & $1.96 \mathrm{E}(-08)$ \\
& N2PC6 & 41 & 0 & 155 & $2.69 \mathrm{E}(-09)$ & $3.25 \mathrm{E}(-10)$ \\
\hline \multirow{2}{*}{$2^{-10}$} & 3ABM6 & 101 & 0 & 240 & $3.34 \mathrm{E}(-09)$ & $3.74 \mathrm{E}(-10)$ \\
& N2PC6 & 62 & 0 & 245 & $9.46 \mathrm{E}(-10)$ & $1.75 \mathrm{E}(-10)$ \\
\hline
\end{tabular}




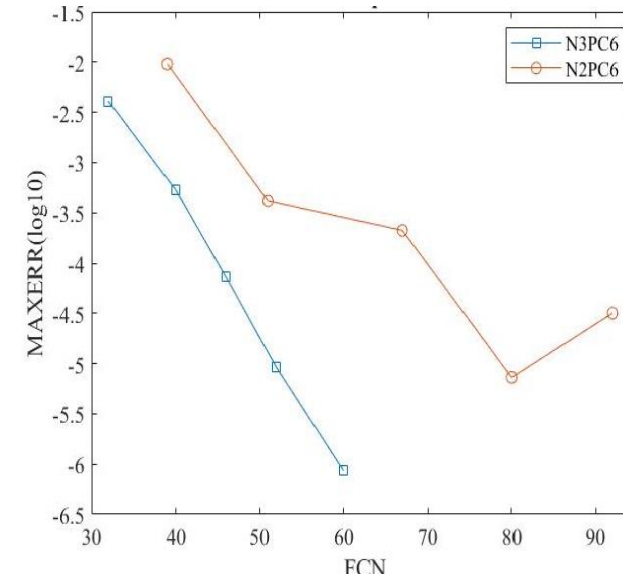

Fig 2: Numerical Results Comparison of Problem 1.

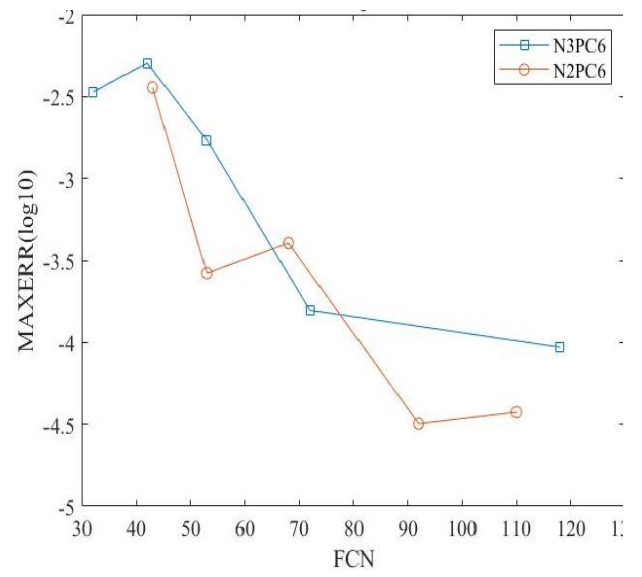

Fig 3: Numerical Results Comparison of Problem 2.

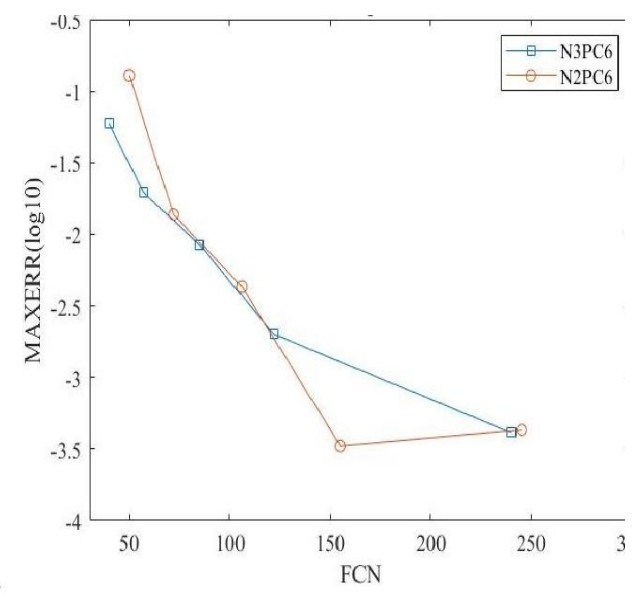

Fig 4: Numerical Results Comparison of Problem 3.

\section{Discussion and Conclusion}

For problem 1, the total number of function calls and the maximum errors obtained by the modified 3-point Adams block method of order six are less than the 2-point order six PC method. For problem 2 , the total number of function calls is less than the N2PC method, except for tolerance $10^{-10}$ which is slightly high. Also, for problem 3, the total number of function calls is less than the N2PC method. However, for the given tolerances, the maximum errors for problems 1-3 are within the acceptable range, so both methods achieved the desired accuracy. It is clear from the approximate results that the proposed method is more efficient than the 2-point order six predictor-corrector method in solving NDDEs to the desired accuracy. Figures 2-4 represents the approximate solutions graphically of the problems 13. Our new proposed algorithm is efficient because during the iteration process the total number of function calls are less than the other algorithms used in literature.

In this paper, we have developed a modified 3-point Adams block method of order six based on the variable step size strategy for first-order neutral delay differential equations. The coding of the $\mathrm{C}$ programming language has been used to test the three numerical problems. The results obtained of the three problems tested indicate that the proposed method is more efficient and accurate in terms of the total number of function calls and maximum errors than the 2-point order six predictor-corrector method.

\section{REFERENCES}

[1] K. W. Neves, "Automatic integration of functional differential equations:," an approach, ACM Trans. Math. Softw. 1, pp. 357-368, 1975.

[2] A. Bellen and M. Zennaro, "Numerical solution of delay differential equations by uniform corrections to an implicit Runge- 
Kutta method" Numer Math, vol. 47, no. 2, pp. 301-316, 1985

[3] A. N. Al-Mutib, "Stability properties of numerical methods for solving delay differential equations," J. Comput. Appl. Math., vol. 10, no. 1, pp. 71-79, 1984.

[4] Z. Jackiewicz and E. Lo, "The numerical solution of neutral functional differential equations by Adams predictor- corrector methods," Appl. Numer. Math., vol. 8, no. 6, pp. 477-491, 1991.

[5] L. Tavernini, "Linear Multistep Methods for the Numerical Solution of Volterra Functional Differential Equations," Appl. Anal., vol. 3, no. 2, pp. 169-185, 1973.

[6] R. H. Fabiano and C. Payne, "Spline approximation for systems of linear neutral delay-differential equations," Appl. Math. Comput., vol. 338, pp. 789-808, 2018.

[7] H. Qin, Q. Zhang, and S. Wan, "The continuous Galerkin finite element methods for linear neutral delay differential equations," Appl. Math. Comput., vol. 346, no. 11501514 , pp. 76-85, 2019.

[8] L. Wen, X. Liu, and Y. Yu, "Stability of Runge-Kutta Methods for Neutral Delay Differential Equations," Discret. Dyn. Nat. Soc., vol. 2015, no. January 2015, 2015.

[9] C. T. H. Baker, G. A. Bocharov, and F. A. Rihan, "Neutral Delay Differential Equations in the Modelling of Cell Growth," J. Egypt. Math. Soc., vol. 16, no. 2, pp. 133160, 2008.

[10] P. Hu, C. Huang, and S. Wu, "Asymptotic stability of linear multistep methods for nonlinear neutral delay differential equations," Appl. Math. Comput., vol. 211, no. 1, pp. 95-101, 2009.

[11] P. Hu and C. Huang, "Analytical and numerical stability of nonlinear neutral delay integro-differential equations," J. Franklin Inst., vol. 348, no. 6, pp. 1082-1100, 2011.

[12] W. S. Wang and S. F. Li, "On the one-leg $\theta$-methods for solving nonlinear neutral functional differential equations," Appl. Math. Comput., vol. 193, no. 1, pp. 285301, 2007.

[13]H. Y. Seong and Z. A. Majid, "Solving second order delay differential equations using direct two-point block method," Ain Shams Eng. J., vol. 8, no. 1, pp. 59-66, 2017.
[14]N. H. A. Aziz and Z. A. Majid, "In Press , Accepted Manuscript - Note to users The Technique of Discontinuity Tracking Equations for Functional Differential Equations in 1-Point Implicit Method In Press , Accepted Manuscript - Note to users."

[15] S. O. Fatunla, "Block methods for second order odes," Int. J. Comput. Math., vol. 41, no. 1-2, pp. 55-63, 1991.

[16]F. Ishak, Z. A. Majid, and M. B. Suleiman, "Efficient interpolators in implicit block method for solving delay differential equations," Int. J. Math. Comput. Simul., vol. 7 , no. 2 , pp. 116-124, 2013.

[17]M. Alkasassbeh and Z. Omar, "Generalized Hybrid One-Step Block Method Involving Fifth Derivative for Solving Fourth-Order Ordinary Differential Equation Directly," J. Appl. Math., vol. 2017, 2017.

[18] J. D. Lambert, "Computational Methods in Ordinary Differential Equations. London," London: Wiley, 1973.

[19]Z. Abdul Majid and M. Suleiman, "Predictor-corrector block iteration method for solving ordinary differential equations," Sains Malaysiana, vol. 40, no. 6, pp. 659664, 2011.

[20]Z. Jackiewicz, "Numerische mathematik Adams methods for neutral functional," Differ. Equations, vol. 230, pp. 221-230, 1982. 